\title{
Formal demonstration of the independence on the optical activity of the maximum gain position in a two-wave coupling experiment
}

\author{
Jorge A. Gómez, ${ }^{* 1}$ and Ángel Salazar \\ Grupo de Óptica y Espectroscopía (GOE), Centro de Ciencia Básica, Universidad Pontificia Bolivariana, \\ A.A. 56006, Medellín, Colombia, \\ ${ }^{1}$ Grupo de Física Básica y Aplicada, Politécnico Colombiano Jaime Isaza Cadavid, Medellín, Colombia
}

Received November 14, 2010; accepted December 15, 2010; published December 31, 2010

\begin{abstract}
The two-beam coupling method has been a useful procedure to estimate the screening Debye length in a photorefractive crystal. The position and value of the maximum gain value as a function of the photorefractive spacing grating is used to obtain the Debye length value. This position and not the maximum value of the gain, appears to be evidently independent of the optical activity of the material. However, a formal treatment to this effect is not found in the specialized literature. In this paper, we present such formal treatment.
\end{abstract}

Holographic techniques using photorefractive crystals have been demonstrated to be appropriated for the characterization of crystals themselves. In particular, a two-beam coupling constitutes a suitable technique for material characterization. Direct holographic techniques and phase modulation techniques for self-stabilized recording and fringe-locked holograms have been used to measure some of the most important parameters [1]-[21]. Recently, in Ref. [1], a procedure for the simultaneous determination of the screening Debye length and the electro-optic coefficient for an optically active $\mathrm{Bi}_{12} \mathrm{SiO}_{20}$ (BSO) crystal, has been presented. For the computation of these material parameters, experimental data of gain vs. grating spacing have been obtained by a two-beam arrangement. A general expression for the diffraction efficiency in the presence of self-diffraction was considered. The fitting of the experimental data to the predicted theoretical behavior was achieved by finding the closest theoretical curve to a set of data sampled from a spline-smoothed curve of the experimental data. Both the Debye screening length $l_{\mathrm{s}}$ and the electro-optic coefficient $r_{41}$ were used as fitting parameters. By searching in wide intervals for each one of the parameters, the estimation of their values was obtained in a reliable and direct way from only one experiment. Calculations were performed in the diffusion regimen and the procedure led to $l_{\mathrm{s}}=0.22 \mu \mathrm{m}$ and $r_{41}=4.5 \cdot 10^{-12} \mathrm{~m} / \mathrm{V}$. Because the optical activity can alter the maximum gain value, and self-diffraction effects influence the energy exchange, the procedure turned out to be physically appropriated for the simultaneous determination of these physical parameters when thick photorefractive crystals with high optical activity were used (in our experimental set up, the crystal depth was $L=10 \mathrm{~mm}$ ). The result of the fitting was shown in Fig. 4(b) of Ref. [1]. The effect of the optical activity is to reduce the gain. Figure $5(\mathrm{~b})$ in Ref. [1] shows the graphs for the coupling factors $\Gamma L$ and $\bar{\Gamma} L[2]$ as functions of the crystal thickness, with and without optical activity, respectively. The curves show that coupling factors coincide for thin crystals $(L \leq 1 \mathrm{~mm})$, where the effect of optical activity is negligible. However, for thick crystals $(L>1 \mathrm{~mm})$, the difference is notable. The error in the calculation of the theoretical gain would considerably increase for an optically active thick crystal if its effect is not taken into account. Figure 1 shows the theoretical curves of gain using our treatment for a $10 \mathrm{~mm}$ BSO crystal, maintaining the obtained parameters $l_{\mathrm{s}}=0.22 \mu \mathrm{m}$ and $r_{41}=4.5 \cdot 10^{-12} \mathrm{~m} / \mathrm{V}$ fixed, but modifying the optical activity from its value $\rho=37^{\circ} / \mathrm{mm}$ measured for our crystal. It is observed that for a given crystal thickness the gain is sensibly affected by the optical activity value.

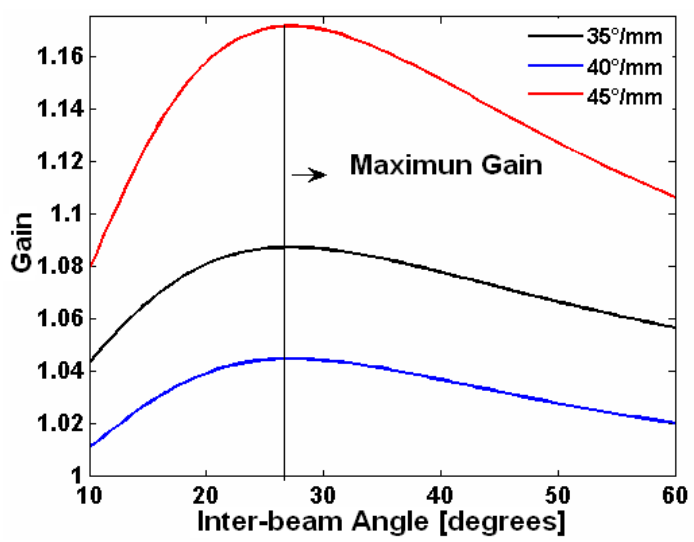

Fig. 1. Theoretical curves of gain maintaining fixed the obtained parameters $l_{\mathrm{s}}$ and $r_{41}$, but theoretically modifying the optical activity from its value $\rho=37^{\circ} / \mathrm{mm}$.

*E-mail: jagomez@elpoli.edu.co 
The maximum of the curve varies depending on the optical activity value. However, as can be seen from Fig. 5(b) in Ref. [1], due to the oscillatory behavior of the coupling factor $\bar{\Gamma} L$ as a function of $L$, a low optical activity does not necessarily means high gain for a given $L$. It must be noticed, however, that the position of the maximum does not appear to be affected by optical activity. It is coherent with the fact that the Debye screening length and optical power are physically independent photorefractive parameters. In this way, even in an optically active crystal, the maximum gain is obtained when $K l_{\mathrm{s}}=1$ [2]. In our experiment, the maximum gain is obtained for a grating spacing $\Lambda=1.35 \mu \mathrm{m}$, which, from $K l_{\mathrm{s}}=1$, corresponds to $l_{\mathrm{s}}=0.22 \mu \mathrm{m}$.

The purpose of this paper is to illustrate by formal treatment that the position of the maximum is not affected by optical activity in a gain vs. a spacing grating set of data. Changing the factor $\Gamma L$ by $\bar{\Gamma} L$ in Eq. (19) of Ref. [1], according to Eq. (19) in the same reference, it is obtained that

$$
G=\frac{I_{S}(L)}{I_{S}^{o}(L)} \approx \exp (\bar{\Gamma} L)=\exp \left\{ \pm \frac{4 \pi \sin \phi_{p}}{\lambda \cos \theta} \frac{\left|\Delta n_{x}\right|}{2}\left[L+\frac{1}{\rho} \cos (2 \varphi+\rho L) \sin (\rho L)\right]\right\}
$$

where, $I_{S}(L)$ and $I_{S}^{0}(L)$ are the output signal intensities when the pump beam is switched on and switched off, respectively; $\phi_{p}$ is the holographic phase; $\Delta n_{x}$ is the index modulation in the crystallographic configuration $<001>$; $\theta$ is the half inter-beam angle; $\lambda$ is the laser wavelength; $\rho$ is the optical activity; $\varphi$ is the input beam polarization angle; and $L$ is the crystal thickness.

Differentiating this expression with respect to the magnitude of the grating vector $K$,

$$
\begin{aligned}
\frac{\partial G}{\partial K}= & \left\{ \pm \frac{4 \pi \sin \phi_{p}}{\lambda \cos \theta} \frac{1}{2}\left[L+\frac{1}{\rho} \cos (2 \varphi+\rho L) \sin (\rho L)\right] \frac{\partial\left|\Delta n_{x}\right|}{\partial K}\right\} * \\
& \exp \left\{ \pm \frac{4 \pi \sin \phi_{p}}{\lambda \cos \theta} \frac{\left|\Delta n_{x}\right|}{2}\left[L+\frac{1}{\rho} \cos (2 \varphi+\rho L) \sin (\rho L)\right]\right\}
\end{aligned}
$$

A maximum of gain is obtained when $\partial G / \partial K=0$. Then, considering that the exponential factor is never zero, we get

$$
\pm \frac{4 \pi \sin \phi_{p}}{\lambda \cos \theta} \frac{1}{2}\left[L+\frac{1}{\rho} \cos (2 \varphi+\rho L) \sin (\rho L)\right] \frac{\partial\left|\Delta n_{x}\right|}{\partial K}=0
$$

The experimental conditions (see Ref. [1]) prevent that the term multiplying the derivative of $\left|\Delta n_{x}\right|$ is zero. Therefore, it follows that $\partial\left|\Delta n_{x}\right| / \partial K=0$. Now, writing $\left|\Delta n_{x}\right|=0.5 r_{41} n^{3}\left|E_{\text {eff }}\right|$ and taking into account that the magnitude of the effective field in absence of external electric field is given by $\left|E_{\text {eff }}\right|=\operatorname{Im}\left\{\left|E_{\text {eff }}\right|\right\}=E_{D} /\left(1+K^{2} l_{s}{ }^{2}\right)$, the maximum of gain is found when $K l_{\mathrm{s}}=1$. This result demonstrates that the gain maximum in the presence of optical activity is given at the same space grating period than in the case of no optical activity. The maximum gain is given by,

$$
G_{M}=\exp \left\{ \pm \frac{\pi \sin \phi_{p} k_{B} T}{\lambda e l_{s} \cos \theta}\left[L+\frac{1}{\rho} \cos (2 \varphi+\rho L) \sin (\rho L)\right]\right\}
$$

or

$$
G_{M}=\exp \left\{ \pm \frac{\pi \sin \phi_{p}{ }^{k} B^{T}}{\lambda e l_{S} \cos \theta} L[1+\cos (2 \varphi+\rho L) \operatorname{sinc}(\rho L)]\right\}
$$

where, $k_{B}$ is the Boltzman constant; $T$ is the absolute temperature; $e$ is the magnitude of the electron charge. The diffusion field has been written in terms of $l_{\mathrm{s}}$ by using the result $K l_{\mathrm{s}}=1$. From the last expressions it is clear that for a given crystal depth $L$, the maximum gain depends on the optical activity and its value fluctuates because of the oscillating characteristic of the functions cos and sinc. A similar conclusion is obtained for a given optical activity if the crystal thickness is modified, as shown in Fig. 2.

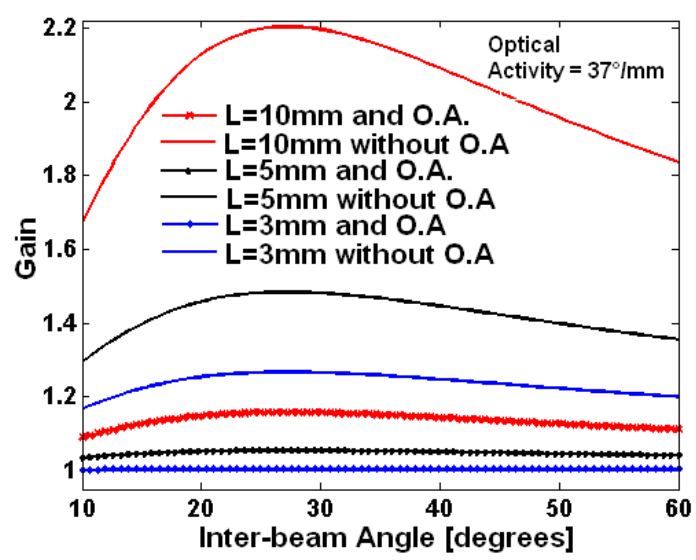

Fig. 2. Theoretical curves of gain maintaining fixed the obtained parameters $l_{s}$ and $r_{41}$ in Ref. [1] for zero optical activity (O.A) or for $\rho=37^{\circ} / \mathrm{mm}$. The crystal thickness $L$ is theoretically modified.

In conclusion, the two-beam coupling method constitutes a useful procedure to estimate the screening Debye length in a photorefractive crystal. The procedure is based on the position of maximum gain for a gain vs. spacing grating set of data. Recently, in Ref. [1], the method has been used, together with a numerical technique, to simultaneously determinate the Debye length and the electro-optic coefficient of a BSO crystal. The procedure does not depend on the position of maximum gain. However, and in spite of that, formal treatment is not found in the literature to demonstrate that the position of the maximum in a gain vs. spacing grating 
set of data in an optically active photorefractive crystal is not affected by optical activity. The purpose of this paper has been to offer this formal treatment. For the best understanding of the paper, Ref. [1] should be followed.

J. A. Gómez acknowledges the financial support of the Politécnico Colombiano Jaime Isaza Cadavid for his Ph.D. studies and for the resources for the research project 2061090125. A. Salazar acknowledges the financial support of the Universidad Pontificia Bolivariana, Medellín-Colombia, and its Centro de Investigación para el Desarrollo y la Innovación, CIDI, through the research project 392A-12/08-27.

\section{References}

[1] J.A. Gómez, H. Lorduy, Á. Salazar, Opt. Commun. 284, 460 (2011).

[2] J. Frejlich, Photorefractive Materials, Fundamental Concepts, Holographic recording and Materials Characterization (John Wiley \& Sons, Inc. USA 2007).
[3] R.W. Mullen, in: P. Günter and J.-P. Huignard (Eds.), Photorefractive Materials and Their Applications I, Fundamental Phenomena (Springer-Verlag, Berlin 1988).

[4] P.A.M. dos Santos, P.M. Garcia, J. Frejlich, J. Appl. Phys. 66, 247 (1989).

[5] L. Arizmendi, J. Appl. Phys. 64, 4654 (1988).

[6] P. M. Garcia, L. Cescato, J. Frejlich, J. Appl. Phys. 66, 47 (1989)

[7] J. Frejlich, P. M. Garcia, L. Cescato, Opt. Lett. 14, 1210 (1989).

[8] J. Frejlich, P. M. Garcia, L. Cescato, Cescato, Opt. Lett. 15, 1247 (1990).

[9] D.J. Webb, L. Solymar, Opt. Commun. 83, 287 (1991).

[10] S. Bian, J. Frejlich, J. Opt. Soc. Am. B. 12, 2060 (1995).

[11] A.A. Freschi, P.M. Garcia, J. Frejlich, Appl. Phys. Lett. 71, 2247 (1997).

[12] I. de Oliveira, J. Frejlich, Opt. Commun. 178, 251 (2000).

[13] I. de Oliveira, J. Frejlich, J. Opt. Soc. Am. B 18, 291 (2001).

[14] L. Mosquera, I. de Oliveira, J. Frejlich, J. Appl. Phys. 90, 2635 (2001).

[15] I. de Oliveira, J. Frejlich, Phys. Ver. A 64, 033806 (2001).

[16] M.C. Barbosa, L. Mosquera, J. Frejlich, Appl. Phys. B, 72, 717 (2001).

[17] M.C. Barbosa, J. Frejlich, J. Opt. A: Pure Appl. Opt. 5, S416 (2003).

[18] I. de Oliveira, J. Frejlich, J. Opt. A: Pure appl. Opt. 5, S428 (2003).

[19] R. Montenegro, A.A. Freschi, J. Frejlich, J. Opt. A.: Pure Appl. Opt. 10, 104006 (2008)

[20] A. Salazar, H. Lorduy G., R. Montenegro, J. Frejlich, J. Opt. A: Pure appl. Opt. 11, 1 (2009).

[21] A. Shumelyuk, M. Wesner, M. Imlau, S. Odoulov, Appl. Phys. B 95, 497 (2009). 